\title{
A RECONNAISSANCE OF THE POSSIBLE DONOR STARS TO THE KEPLER SUPERNOVA
}

\author{
Wolfgang E. Kerzendorf ${ }^{1,2}$, Michael Childress ${ }^{1}$, Julia Scharwächter ${ }^{3}$, Tuan Do ${ }^{2}$, And Brian P. Schmidt ${ }^{1}$ \\ ${ }^{1}$ Research School of Astronomy and Astrophysics, Mount Stromlo Observatory, Cotter Road, \\ Weston Creek, ACT 2611, Australia; wkerzend@mso.anu.edu.au \\ ${ }^{2}$ Department of Astronomy and Astrophysics, University of Toronto, 50 Saint George Street, Toronto, ON M5S 3H4, Canada \\ ${ }^{3}$ Observatoire de Paris, LERMA (CNRS: UMR 8112), 61 Av. de l'Observatoire, 75014 Paris, France \\ Received 2013 July 8; accepted 2013 September 15; published 2014 January 23
}

\begin{abstract}
The identity of Type Ia supernova progenitors remains a mystery, with various lines of evidence pointing toward either accretion from a nondegenerate companion or the rapid merger of two degenerate stars leading to the thermonuclear destruction of a white dwarf. In this paper, we spectroscopically scrutinize 24 of the brightest stars residing in the central $38^{\prime \prime} \times 38^{\prime \prime}$ of the SN 1604 (Kepler) supernova remnant to search for a possible surviving companion star. We can rule out, with high certainty, a red giant companion star-a progenitor indicated by some models of the supernova remnant. Furthermore, we find no star that exhibits properties uniquely consistent with those expected of a donor star down to $L>10 L_{\odot}$. While the distribution of star properties toward the remnant are consistent with unrelated stars, we identify the most promising candidates for further astrometric and spectroscopic follow up. Such a program would either discover the donor star or place strong limits on progenitor systems to luminosities with $L \ll L_{\odot}$.
\end{abstract}

Key words: ISM: supernova remnants - supernovae: individual (SN1604)

Online-only material: color figures

\section{INTRODUCTION}

Type Ia supernovae ( $\mathrm{SNe}$ Ia) provide a means to measure cosmological distances and are also major contributors to the chemical evolution of the universe, enriching the cosmos with large amounts of iron-peak elements. Despite considerable effort over the past decade to identify the systems that become SN Ia, no consensus has yet emerged. It is widely accepted that $\mathrm{SNe}$ Ia are the thermonuclear explosions of massive carbon/oxygen white dwarves (CO-WD). The community suggests two main progenitor scenarios (for a review see Wang \& Han 2012 and references therein). In the first scenario, the WD accretes mass from a non-degenerate companion (known as a donor star) until the central temperature and density exceed the cooling threshold and run-away nuclear burning occurs. This is usually described as the single-degenerate scenario (SD-scenario). In the second scenario, two WDs merge, leading to a cataclysmic explosion (double-degenerate scenario; DD-scenario).

Both events make a number of predictions, however, only the SD-scenario offers a directly observable prediction-the remaining donor star. At first glance, the SD-scenario looks easily falsifiable. The donor star should remain (e.g., Marietta et al. 2000) and be easy to detect in historical remnants. There has been considerable effort (Ruiz-Lapuente et al. 2004; González Hernández et al. 2009, 2012; Kerzendorf et al. 2009, 2012a, 2012b; Schaefer \& Pagnotta 2012; Edwards et al. 2012) to find the donor stars of ancient supernovae. Two remnants in the LMC (Edwards et al. 2012; Schaefer \& Pagnotta 2012) and SN 1006 (Kerzendorf et al. 2012a; González Hernández et al. 2012) have proven to be void of progenitors to a relatively stringent detection limit. A possible progenitor has been found in SN 1572 (Ruiz-Lapuente et al. 2004), although the validity of this connection has been challenged (Kerzendorf et al. 2012b). Despite these negative results, this does not necessarily disprove the validity of the SD-scenario, as one can construct scenarios for the single-degenerate case that produce surviving companions that do not stand out among their neighboring stars (e.g., Pan et al. 2012; Liu et al. 2013; Podsiadlowski 2003).

The recent discoveries of circumstellar medium (CSM) around roughly a quarter of normal SNe Ia (Patat et al. 2007; Simon et al. 2009; Sternberg et al. 2011; Foley et al. 2012) seemingly suggests two subtypes. Since CSM interaction is favored by the SD-scenario and is more rare, the lack of the direct detection of a donor star could just be due to the relative rarity of these objects.

In this work, we turn to the last unexamined (in respect to donor stars) young Galactic supernova remnant - the remnant of SN 1604 (also known as Kepler's SN). There is a dispute about the precise distance $(4-6.4 \mathrm{kpc}$; see Chiotellis et al. 2012 and references therein), and we choose to adopt the upper and more conservative limit of $d=6.4 \mathrm{kpc}$ (Reynoso \& Goss 1999) for this work. Furthermore, there is a known maximum apparent brightness $(V=-3$; Pskovskii 1971), which, together with a usual absolute brightness of SNe Ia $\left(M_{V}=-19.3\right.$; Riess et al. 2011) and an extinction of $A_{V}=$ 2.8 (Reynolds et al. 2007), leads to a distance of $\approx 5 \mathrm{kpc}$, consistent with the other measurements. Using the historic brightness provides an independent and direct measurement of distance modulus and extinction, which proves useful when testing the candidates' luminosities. Compared to its Galactic siblings (SN 1006 and SN 1572), the Kepler SNR shows a very unusual structure and the presence of a nitrogen-rich shell and therefore had initially been identified as a core collapse (e.g., Bandiera 1987). However, more recent studies affirm its SN Ia nature, primarily due to its large amount of $\mathrm{Fe}$ and little oxygen (Reynolds et al. 2007; Patnaude et al. 2012). Chiotellis et al. (2012) attribute the shell to a progenitor with high mass loss prior to explosion - a calling card for the SD-scenario. This large amount of interstellar matter suggests that Kepler might have belonged to the class of SNe Ia showing CSM interaction in their spectra. Finally, the remnant shows a high systemic velocity of $250 \mathrm{~km} \mathrm{~s}^{-1}$ (Blair et al. 1991; Sollerman et al. 2003). This high systemic velocity, including a 


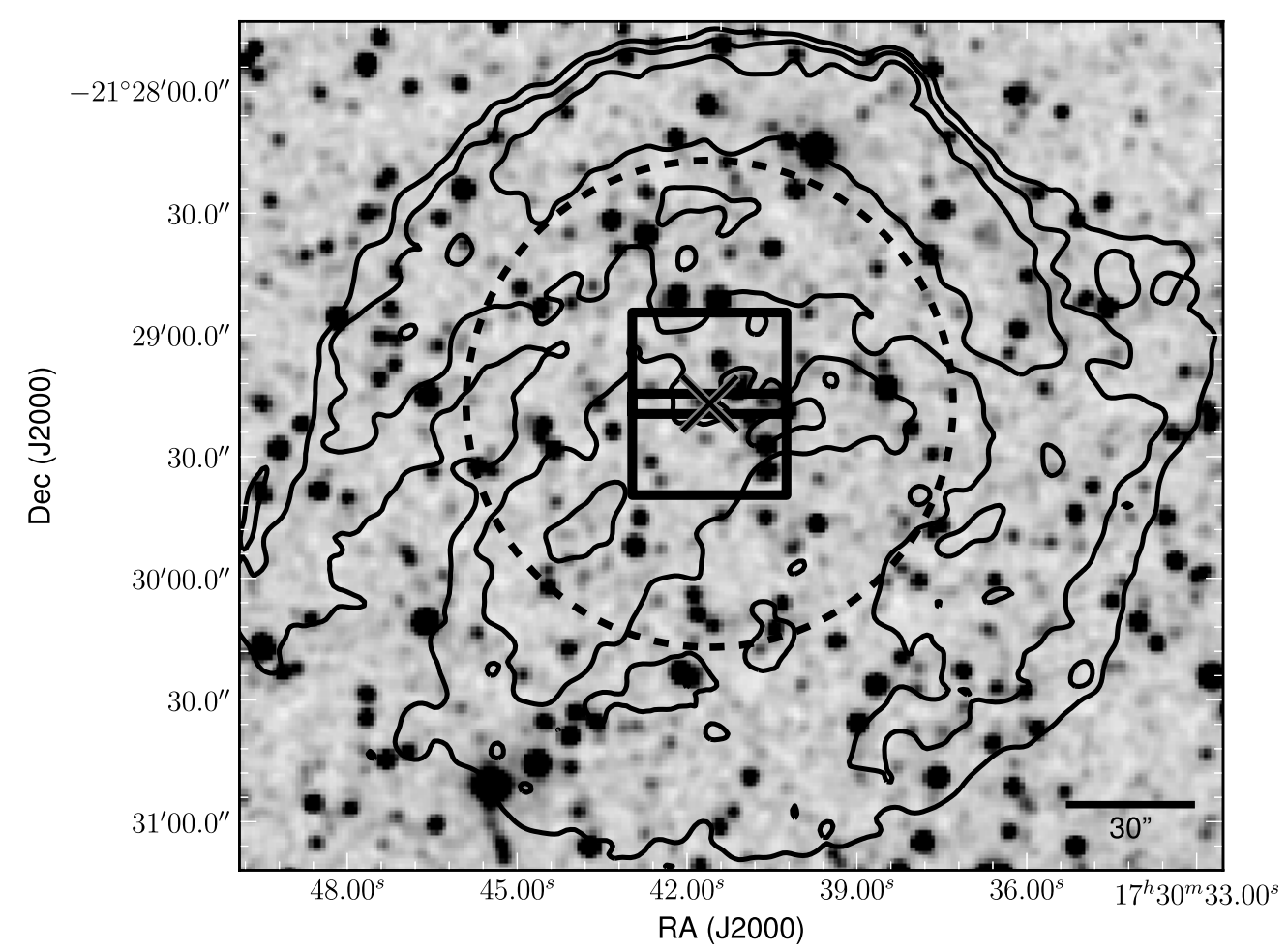

Figure 1. SN1604 Two Micron All Sky Survey (Skrutskie et al. 2006) image with contours of ACIS X-ray image (ObsID 116; Hwang et al. 2002). In this case, 19" equals $1420 \mathrm{~km} \mathrm{~s}^{-1}$ at $6.4 \mathrm{kpc}$ over $400 \mathrm{yr}$. The dashed circle describes a $60^{\prime \prime}$ circle where we photometrically searched for potential companion stars.

radial velocity component of $-180 \mathrm{~km} \mathrm{~s}^{-1}$, should help make it possible to more easily identify a potential donor star, as any star associated with the SN should have $-180 \mathrm{~km} \mathrm{~s}^{-1}$ of motion added to its orbital motion at the time of the explosion.

Here, we report a photometric and spectroscopic search for a donor star in the SN 1604 remnant. In Section 2, we give an overview of the observation of the spectroscopic data and describe the Hubble Space Telescope (HST) data. We describe the analysis of the spectroscopic data in Section 3 and discuss its implications in Section 4. We conclude this paper and discuss possible future work in Section 5.

\section{OBSERVATIONS}

\subsection{Integral Field Spectroscopy}

We used the Integral Field Unit called WIFES (Dopita et al. 2007) mounted on the ANU $2.3 \mathrm{~m}$ Telescope at the Siding Spring Observatory to undertake the spectroscopic observations in this study. The WIFES-spectrograph is an image slicer with $2538 \times 1^{\prime \prime}$ slitlets and 0.'5 sampling in the spatial direction on the detector. We chose this instrument for its large field of view $\left(25^{\prime \prime} \times 38^{\prime \prime}\right)$ and a resolution of $R=7000$ to provide the best possible radial velocity measurements.

We have adopted $\alpha=17^{\mathrm{h}} 30^{\mathrm{m}} 41^{\mathrm{s}} .6 \delta=-21^{\circ} 29^{\prime} 17^{\prime \prime}$ as the center of the remnant from Katsuda et al. (2008). Due to the potential uncertainty in the center determination (for a more detailed discussion about the difficulty of center determination see Section 2.2 in Kerzendorf et al. 2012a), we split up our observations to cover two fields of $25^{\prime \prime} \times 38^{\prime \prime}$ with an overlap giving a total field of $38^{\prime \prime} \times 38^{\prime \prime}$ (see Figure 1). This setup gives us a minimum search distance of $19^{\prime \prime}$ from the proposed center, corresponding to a velocity of $1420 \mathrm{~km} \mathrm{~s}^{-1}$ (in the plane of the sky over $400 \mathrm{yr}$ ) assuming a remnant distance of $6.4 \mathrm{kpc}$ (Chiotellis et al. 2012).
We selected the 17000 grating, which covers a spectral region (6830-9120 $\mathrm{A})$ with $0.2 \AA\left(7 \mathrm{~km} \mathrm{~s}^{-1}\right)$ resolution. This region includes the Ca II triplet ( $8498 \AA, 8542 \AA$, and $8662 \AA$ ) and maximizes the signal to noise of the stars observed, which are heavily reddened by dust. The Ca II provides good radial velocity measurements even in low signal-to-noise ratio observations.

The observations were carried out on 2010 June 15 and 16. The exposure time for all observations was $600 \mathrm{~s}$ with the southern field being observed 33 times $(5.5 \mathrm{hr})$ and the northern field observed 19 times $(3.2 \mathrm{hr})$ as weather conditions deteriorated. The seeing ranged between $1^{\prime \prime} .7$ and 2 .'7 with a median of 1".9 (see the left panel of Figure 2 for a sample of the data quality).

WIFES data were processed using the PyWiFeS package. ${ }^{4}$ Wavelength solutions for each data cube were derived from night sky emission lines, achieving a residual wavelength solution scatter of $0.1 \AA$.

In a final step, a simple spatial world coordinate system was applied to each cube with the help of the Two Micron All Sky Survey (2MASS) for object coordinates (as 2MASS shows a similarly broad PSF).

\subsection{Photometry}

To obtain accurate photometry, we used archival $H S T$ data (HST program GO-9731) observed in 2003 August. The data consist of two F550M images with an exposure time of $240 \mathrm{~s}$ each, observed with the Advanced Camera for Surveys (ACS). We analyzed the frames using DOLPHOT (Dolphin 2000) and extracted magnitudes for all stars and corrected it to the $V$ band (the difference in flux between F550M and $\mathrm{V}$ for Vega is $0.12 \mathrm{mag}$ ). Table 1 shows the magnitudes of stars

\footnotetext{
4 http://www.mso.anu.edu.au/pywifes/
} 

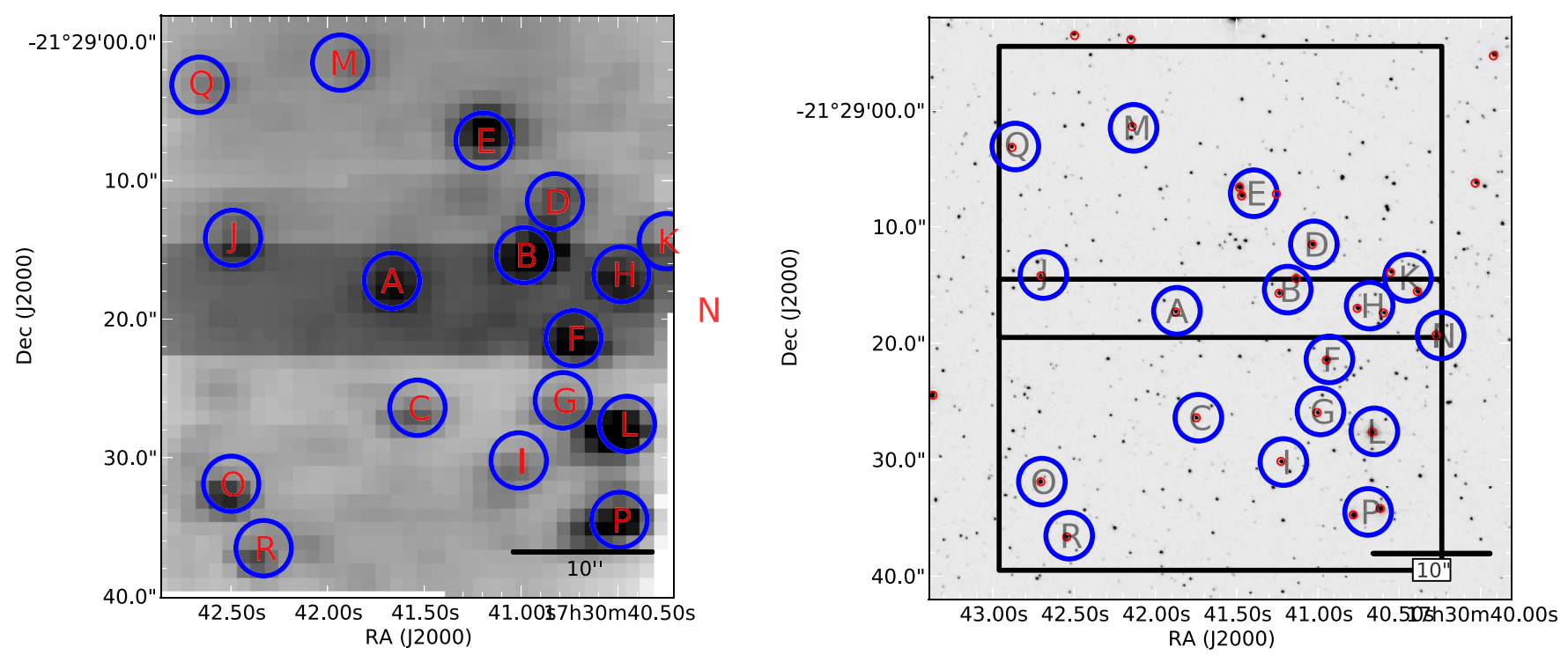

Figure 2. Left panel: the combination of a median (in the spectral direction) of two of our spectral cubes. The extraction regions are marked in blue. The seeing is comparable to the 2MASS, enabling a direct comparison of 2MASS magnitudes and the spectra. Due to dithering, some of the stars described in the analysis (N and $\mathrm{K})$ are not covered by the cubes displayed in this figure. Right panel: HST F550M image of the central of region of SN1604. We have marked the extraction regions with blue circles and the individual sources greater than $V=19.21\left(10 L_{\odot}\right.$ at the distance of the remnant taking the difference between F550M and the $V$ filter into account) with red circles. There are a few extraction regions that contain more than one bright star.

(A color version of this figure is available in the online journal.)

Table 1

HST Measurements of Candidates

\begin{tabular}{|c|c|c|c|c|c|c|c|c|c|c|c|}
\hline $\begin{array}{l}\text { Name } \\
-\end{array}$ & $\begin{array}{c}\text { R.A. } \\
\text { hh:mm:ss.ss }\end{array}$ & $\begin{array}{c}\text { Decl. } \\
\text { dd:mm:ss.s }\end{array}$ & $\begin{array}{c}\text { F550M } \\
\text { (mag) }\end{array}$ & $\begin{array}{c}\operatorname{Luminosity}(V)^{\mathrm{a}} \\
\left(L_{\odot}\right)\end{array}$ & $\begin{array}{c}\text { NOMAD ID } \\
-\end{array}$ & $\begin{array}{c}B^{\mathrm{c}} \\
(\mathrm{mag})\end{array}$ & $\begin{array}{c}V^{\mathrm{c}} \\
(\mathrm{mag})\end{array}$ & $\begin{array}{c}R^{\mathrm{c}} \\
(\mathrm{mag})\end{array}$ & $\begin{array}{c}J^{\mathrm{c}} \\
(\mathrm{mag})\end{array}$ & $\begin{array}{c}H^{\mathrm{c}} \\
(\mathrm{mag})\end{array}$ & $\begin{array}{c}K^{\mathrm{c}} \\
(\mathrm{mag})\end{array}$ \\
\hline $\mathrm{A} 1$ & $17: 30: 41.88$ & $-21: 29: 17.3$ & 18.93 & 9 & $0685-0474466$ & $\ldots$ & $\ldots$ & 17.6 & 15.1 & 14.5 & 14.2 \\
\hline B1 & $17: 30: 41.13$ & $-21: 29: 14.4$ & 17.55 & 33 & $0685-0474416^{\mathrm{b}}$ & 18.0 & 16.7 & 13.9 & 14.0 & 13.2 & 12.9 \\
\hline $\mathrm{B} 2$ & $17: 30: 41.24$ & $-21: 29: 15.7$ & 18.98 & 9 & $0685-0474416^{b}$ & 18.0 & 16.7 & 13.9 & 14.0 & 13.2 & 12.9 \\
\hline $\mathrm{C} 1$ & $17: 30: 41.75$ & $-21: 29: 26.4$ & 18.90 & 9 & $0685-0474460$ & $\ldots$ & $\ldots$ & 17.9 & 15.6 & 14.9 & 14.6 \\
\hline D1 & $17: 30: 41.04$ & $-21: 29: 11.5$ & 18.07 & 20 & 0685-0474416 & 18.0 & 16.7 & 13.9 & 14.0 & 13.2 & 12.9 \\
\hline$E 2^{d}$ & $17: 30: 41.47$ & $-21: 29: 07.3$ & 17.79 & 26 & 0685-0474437 & 17.1 & 16.1 & 15.3 & 13.6 & 12.9 & 12.6 \\
\hline E3 & 17:30:41.26 & $-21: 29: 07.2$ & 18.95 & 9 & 0685-0474437 & 17.1 & 16.1 & 15.3 & 13.6 & 12.9 & 12.6 \\
\hline $\mathrm{F} 1$ & $17: 30: 40.95$ & $-21: 29: 21.5$ & 17.95 & 23 & 0685-0474398 & 18.8 & $\ldots$ & 15.9 & 14.4 & 13.7 & 13.4 \\
\hline G1 & 17:30:41.01 & $-21: 29: 26.0$ & 18.92 & 9 & 0685-0474409 & $\ldots$ & $\ldots$ & $\ldots$ & 15.5 & 14.6 & 14.3 \\
\hline $\mathrm{H} 1$ & $17: 30: 40.76$ & $-21: 29: 17.0$ & 18.69 & 11 & 0685-0474388 & $\ldots$ & $\ldots$ & $\ldots$ & 15.0 & 14.2 & 14.0 \\
\hline $\mathrm{H} 2$ & $17: 30: 40.60$ & $-21: 29: 17.4$ & 19.16 & 7 & 0685-0474388 & $\ldots$ & $\ldots$ & $\ldots$ & 15.0 & 14.2 & 14.0 \\
\hline I1 & $17: 30: 41.23$ & $-21: 29: 30.1$ & 19.18 & 7 & 0685-0474414 & 20.3 & $\ldots$ & 17.1 & $\ldots$ & $\ldots$ & $\ldots$ \\
\hline $\mathrm{K} 1^{\mathrm{d}}$ & $17: 30: 40.39$ & $-21: 29: 15.6$ & 17.91 & 23 & $0685-0474365$ & $\ldots$ & 16.4 & $\ldots$ & 14.8 & 14.2 & 14.1 \\
\hline $\mathrm{K} 2$ & $17: 30: 40.56$ & $-21: 29: 13.9$ & 19.24 & 7 & $0685-0474365$ & $\ldots$ & 16.4 & $\ldots$ & 14.8 & 14.2 & 14.1 \\
\hline $\mathrm{L} 1^{\mathrm{d}}$ & $17: 30: 40.67$ & $-21: 29: 27.6$ & 16.50 & 86 & 0685-0474384 & 17.4 & 15.9 & 12.8 & 13.1 & 12.3 & 12.0 \\
\hline M1 & $17: 30: 42.14$ & $-21: 29: 01.4$ & 19.16 & 7 & 0685-0474479 & .. & $\ldots$ & 18.0 & 15.8 & 15.2 & 14.8 \\
\hline $\mathrm{N} 1^{\mathrm{d}}$ & $17: 30: 40.27$ & $-21: 29: 19.3$ & 17.38 & 38 & 0685-0474362 & 18.5 & 16.2 & $\ldots$ & 14.0 & 13.2 & 13.0 \\
\hline O1 & 17:30:42.71 & $-21: 29: 31.9$ & 17.94 & 23 & 0685-0474522 & 18.7 & 17.7 & 16.7 & 14.7 & 13.9 & 13.6 \\
\hline $\mathrm{P} 1$ & $17: 30: 40.62$ & $-21: 29: 34.2$ & 17.44 & 36 & $0685-0474383$ & 18.1 & 16.3 & $\ldots$ & 13.7 & 13.0 & 12.7 \\
\hline $\mathrm{P} 2$ & $17: 30: 40.78$ & $-21: 29: 34.7$ & 17.89 & 24 & 0685-0474383 & 18.1 & 16.3 & $\ldots$ & 13.7 & 13.0 & 12.7 \\
\hline Q1 & $17: 30: 42.88$ & $-21: 29: 03.2$ & 18.55 & 13 & 0685-0474537 & $\ldots$ & $\ldots$ & 17.8 & 15.8 & 14.9 & 14.9 \\
\hline $\mathrm{R} 1$ & $17: 30: 42.54$ & $-21: 29: 36.6$ & 18.77 & 11 & 0685-0474505 & $\ldots$ & $\ldots$ & 17.2 & 15.3 & 14.5 & 14.4 \\
\hline
\end{tabular}

\section{Notes.}

a Assuming the candidate to be at the distance of SN1604 by using the distance modulus calculated from SN 1604's apparent magnitude and average SNe Ia absolute magnitude, we obtain $m_{V}-M_{V}=16.3$. If we instead assumed a distance of $6 \mathrm{kpc}$ and an extinction of $A_{V}=2.8$, the luminosity increases by a factor of $1.4\left(m_{V}-M_{V}=16.7\right)$.

${ }^{\mathrm{b}}$ In addition to stars marked with the same letter (but different digit), the extraction Regions B and D are also covered by one NOMAD ID.

${ }^{\mathrm{c}}$ Photometry from the NOMAD catalogue (accuracy $\approx 0.3 \mathrm{mag}$ ).

${ }^{\mathrm{d}}$ Candidate for follow up $\left(L>20 L_{\odot}\right.$ and $P\left(\right.$ donor $\left.\left.\mid v_{\text {rad }}\right)>1 \%\right)$. 
with more than 10 solar luminosities in the $V$ band at the distance of the remnant. In addition, we have supplemented the HST photometry of these objects with photometric information from the Naval Observatory Merged Astrometric Data set (NOMAD; Zacharias et al. 2005). Furthermore, Figure 2 (right panel) shows the possible companion candidates and an overlay of the WIFES-fields on the HST image.

\section{ANALYSIS}

Using the HST images as a template, we selected extraction regions in our spectral cubes with an aperture of $2^{\prime \prime}$, a compromise between using the available signal and avoiding contamination from close stars. Despite the small aperture, some extraction regions contain two or three stars. The aperture was then summed at each spectral channel. A sky measurement was obtained in a similar fashion using an annulus with an inner radius of $4^{\prime \prime}$ and an outer radius of $6^{\prime \prime}$. To alleviate the contribution from neighboring stars, the individual pixels of the sky annuli were median combined during extraction. We scaled the sky lines of the sky annuli to the sky lines of the spectra to avoid an over- or under-subtraction to best remove any residual background.

We concentrated on the wavelength region 8400-8700 for the next part of the analysis - as this contains the strong Ca II lines which are useful for radial velocity measurements. The sky-frame scale was fit with a minimizer (Powell 1964) simultaneously with a continuum (third-order polynomial) and a model of the sun $\left(T_{\text {eff }}=5780, \log g=4.4\right.$, and $[\mathrm{Fe} / \mathrm{H}]=$ $0.0)$. Although the signal-to-noise ratio is in principle high enough to measure stellar parameters, the uncertainty in the continuum placement due to sky subtraction errors makes the parameter estimations unreliable. In addition, we lack accurate $(0.02 \mathrm{mag})$ color photometry $(B, V$, and $R$ ) of all the objects (some photometry is only available for a blend of objects) to aid in the determination of stellar parameters. The unreliable placement of the continuum does not affect the radial velocity measurements as they only rely on the position and not the depth of lines.

The scaled sky was subtracted from the spectra and the resulting spectra (for each extraction site) were interpolated on a common wavelength grid and summed up. Next, we convolved a solar spectrum to the required resolution and shifted these synthetic spectra between $-400 \mathrm{~km} \mathrm{~s}^{-1}$ and $200 \mathrm{~km} \mathrm{~s}^{-1}$ in a thousand equally spaced steps. We then compared the observed spectra with the set of synthetic spectra (using the root-meansquare technique) and chose the velocity corresponding to the lowest root-mean-square. We subsequently compared each fit with the spectrum and ascertained that the CaII features were clearly visible in the candidate spectrum and coincided with the shifted solar spectrum (see Figure 3). Furthermore, in the cases where HST photometry showed the presence of two stars, we checked the rms fit for secondary minima that arise when there are two distinct sets of spectra. Only Kepler-H showed two clear sets of CaII features in the spectrum as well as in the rms fit (henceforth designated as F1 and F2). Finally, we determined the typical error of the radial velocity to be $\approx 4.5 \mathrm{~km} \mathrm{~s}^{-1}$ (or a tenth of a resolution element) by measuring the radial velocity in individual frames of a few candidates and looking at the resulting distribution.

When compared to model spectra, we find that the resolution and quality of the spectra do not allow for a reliable determination of the rotational velocity to better than $200 \mathrm{~km} \mathrm{~s}^{-1}$ (e.g., see Figure 3). As seen in Figure 2, we obtained spectra of sources down to $V=19.2$ (which corresponds to $L_{V}=10 L_{\odot}$ taking

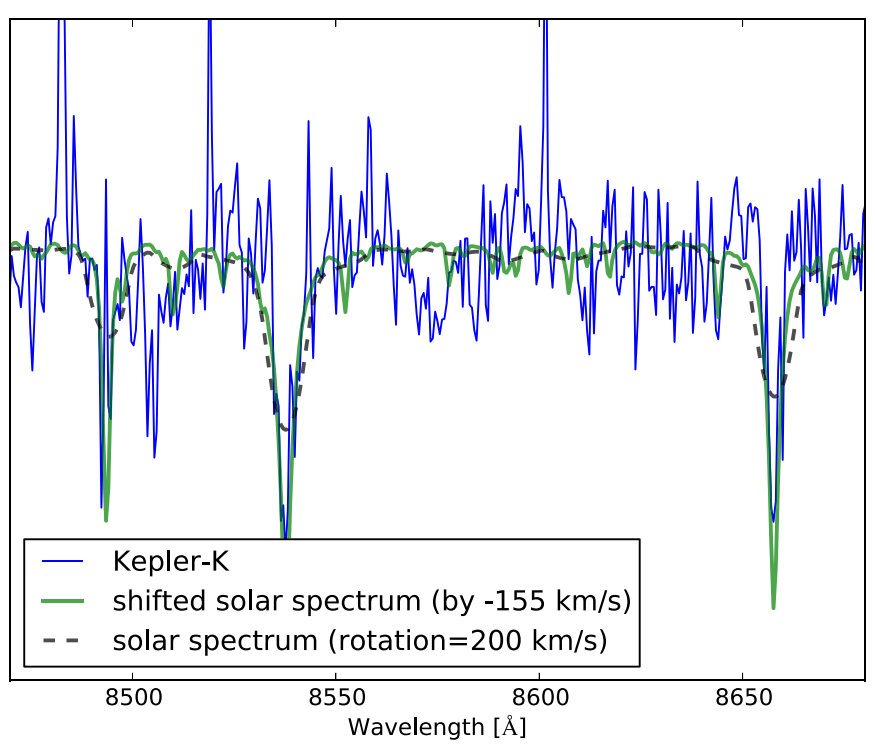

Figure 3. Kepler-K candidate showing a radial velocity of $-155 \mathrm{~km} \mathrm{~s}^{-1}$. Such a velocity is consistent with that expected for more than a third of the donor stars of the Kepler SNR, but also of $5 \%$ of unassociated stars in the direction of the remnant. Given that this study has analyzed 20 stars' radial velocities, this velocity is not, on its own, indicative of an unusual star.

(A color version of this figure is available in the online journal.)

into account distance to the remnant and extinction). However, we could not obtain individual radial velocities for some of the sources that are blended (at $R=7000$ — corresponding to a resolution element width of $42 \mathrm{~km} \mathrm{~s}^{-1}$ - this is not unexpected when examining the radial velocity distribution in Figure 4).

\section{DISCUSSION}

The SD-scenario predicts an escape velocity of the donor of up to $200 \mathrm{~km} \mathrm{~s}^{-1}$ for main-sequence stars and less for sub-giants or giant stars (down to roughly $60 \mathrm{~km} \mathrm{~s}^{-1}$; Han 2008). In the case of SN 1604, this velocity signature might very easily be lost in the kinematic signature of the Galaxy, except that the systemic velocity of the Kepler remnant helps separate candidates from the normal motions of stars along the line of sight. We compare our radial velocity measurements to theoretical predictions by Han (2008) and the Besançon Model (Robin et al. 2003) of galactic dynamics (choosing a $1 \mathrm{deg}^{2}$ area around SN 1604 and limiting to magnitudes between $15<V<20$ ). In Figure 4 , we use a Monte Carlo simulation using the distribution of radial velocities from Han (2008; including main-sequence to giant donors) and a random distribution of ejection angles to obtain a radial velocity distribution. Furthermore, we center this on SNR 1604 's systemic radial velocity of $-185 \mathrm{~km} \mathrm{~s}^{-1}$ (Sollerman et al. 2003). When looking at the Han (2008) distribution in Figure 4, half of the possible cases would produce a star that is a significant outlier compared to the unrelated background and foreground stars. However, none of our stars are significant outliers to the Besançon Model, with our measured radial velocity distribution agreeing very well with the Besançon Model.

We have performed a Bayesian analysis in Table 2 using

$$
\begin{aligned}
P\left(\text { donor } \mid v_{\mathrm{rad}}\right)= & P(\text { donor }) P\left(v_{\mathrm{rad}} ; \text { Han } 2008\right) \\
& /\left(P(\text { donor }) P\left(v_{\mathrm{rad}} ; \text { Han } 2008\right)\right. \\
& \left.+P(\text { not donor }) P\left(v_{\mathrm{rad}} ; \text { Besançon Model }\right)\right)
\end{aligned}
$$

where $P$ (donor) $=1 / 20$ as we assume one star to be the donor star and all other probabilities are determined from the 


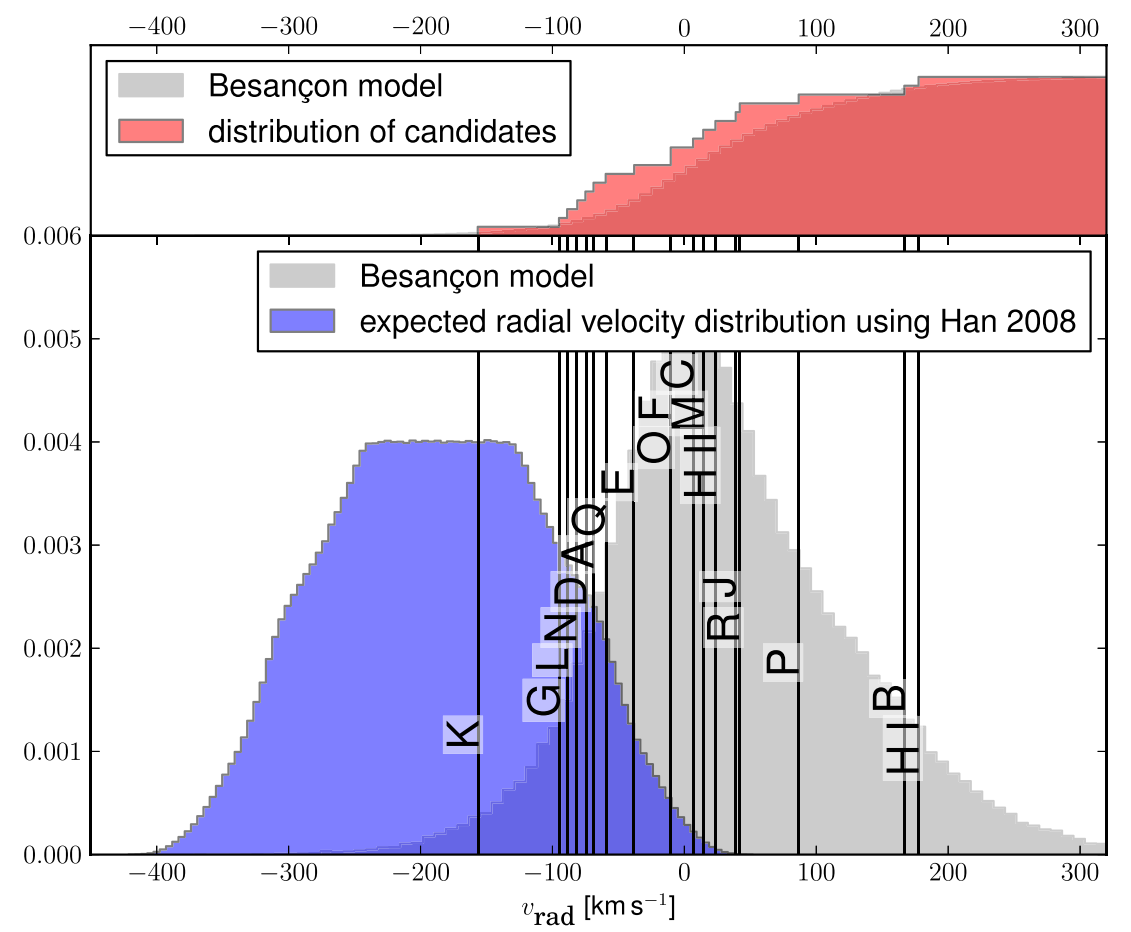

Figure 4. Comparison of Besançon Model (1 deg ${ }^{2}$ around Kepler, V cut between 15 and 20) and Han (2008) and our measured values. The top panel shows the cumulative distribution for the Besançon Model and our measured values. The Han (2008) distribution is the result of a Monte Carlo simulation including the distribution of random angles and two Han (2008) distributions.

(A color version of this figure is available in the online journal.)

Table 2

Radial Velocity Measurements of Candidates ${ }^{\mathrm{a}}$

\begin{tabular}{lrcc}
\hline \hline $\begin{array}{l}\text { Name } \\
\text { Designation }\end{array}$ & $\begin{array}{c}v_{\text {rad }} \\
\left(\mathrm{km} \mathrm{s}^{-1}\right)\end{array}$ & $\begin{array}{c}\text { Distance from Center } \\
(\operatorname{arcsec})\end{array}$ & $\begin{array}{c}P\left(\text { donor } \mid v_{\text {rad }}\right)^{\mathrm{b}} \\
1\end{array}$ \\
\hline $\mathrm{A}$ & -69.07 & 3.75 & 0.05 \\
$\mathrm{~B}$ & 167.17 & 5.99 & 0.00 \\
$\mathrm{C}$ & 7.01 & 9.59 & 0.00 \\
$\mathrm{D}$ & -74.27 & 9.72 & 0.06 \\
$\mathrm{E}$ & -38.64 & 10.29 & 0.02 \\
$\mathrm{~F}$ & -10.51 & 10.33 & 0.01 \\
$\mathrm{G}$ & -94.29 & 12.34 & 0.10 \\
$\mathrm{H} \mathrm{I}^{\mathrm{c}}$ & 177.58 & 12.81 & 0.00 \\
$\mathrm{H} \mathrm{II}^{\mathrm{c}}$ & 23.92 & 12.81 & 0.00 \\
$\mathrm{~J}$ & 39.04 & 15.46 & 0.00 \\
$\mathrm{~K}$ & -155.96 & 16.30 & 0.36 \\
$\mathrm{~L}$ & -88.29 & 16.92 & 0.09 \\
$\mathrm{M}$ & 14.61 & 17.20 & 0.00 \\
$\mathrm{~N}$ & -81.88 & 19.05 & 0.07 \\
$\mathrm{O}$ & -10.71 & 21.37 & 0.01 \\
$\mathrm{P}$ & 86.69 & 21.60 & 0.00 \\
$\mathrm{Q}$ & -59.06 & 22.43 & 0.04 \\
$\mathrm{R}$ & 41.44 & 23.45 & 0.00 \\
\hline
\end{tabular}

\section{Notes.}

${ }^{a}$ We could not reliably measure a radial velocity for Candidate I.

b Probability to be the donor star for the given $v_{\text {rad }}$ and assuming that 1 of 19 must be the donor star.

${ }^{\mathrm{c}}$ Extraction region $\mathrm{H}$ shows two different radial velocities, stemming from the two different stars visible in the $H S T$ image.

probability distributions shown in Figure 4. Kepler-K can be seen as the most anomalous star studied, but using Bayesian analysis we determined that finding an unrelated star that shows a velocity as low or lower than Kepler-K is not unusual given our sample size of 20 stars and the given Besançon Model distribution. However, without additional information, Kepler-K's velocity does not stand-out from the expected velocity distribution for stars along the line of site to Kepler. If we add a prior that 1 of the 19 stars is associated with the remnant, then Kepler-K is the most likely star to be associated (36\% chance-or approximately four times more likely than the next most likely star, Kepler-G).

Marietta et al. (2000) suggests that giant donor stars will be on the order of $1000 L_{\odot}$ for at least $100,000 \mathrm{yr}$ post-explosion. None of the stars in our sample are in that brightness range (the brightest star being Kepler-L with $86 L_{\odot}(V)$ at $6.4 \mathrm{kpc}$ ). Therefore, there seems to be no viable giant donor located in SN 1604. This is evidence against the asymptotic giant branch (AGB) model suggested by Chiotellis et al. (2012) and Burkey et al. (2013), which is one scenario explaining the unusual CSM that surrounds the Kepler remnant, with a 4-5 $M_{\odot}$ AGB donor. Chiotellis et al. (2012) suggest a star with $m_{v}=12.0 \pm 0.5$, which is not present in our candidates.

Williams et al. (2013) have suggested that the center of remnants is not easily determined and suggest widening the progenitor searches. We have used the NOMAD catalog to find bright companions in a $60^{\prime \prime}$ circle around our current center (see Figure 1). The brightest star in the K band reaches $K=10.5$ ( $43^{\prime \prime}$ from the center) and the brightest star in $V$ band reaches $V=$ 14.7 or $L_{V}=330 L_{\odot}$ ( $40^{\prime \prime}$ from the center). Finally, Chiotellis et al. (2012) suggest the possibility of losing the entire envelope and only having a WD left over $\left(0.8 M_{\odot}\right)$. A normal WD would be below our detection limit. However, Marietta et al. (2000) suggest that losing the entire envelope would not result in a normal WD, but a much brighter and hotter object $\left(\approx 10^{3} L_{\odot}\right)$.

For main-sequence and sub-giant companions two new studies (Shappee et al. 2013; Pan et al. 2012) suggest a luminosity 
of $\approx 20-275 L_{\odot}$ for companion stars $400 \mathrm{yr}$ after the explosion (see Figure 6 in Pan et al. 2012). Restricting ourselves to objects that have $\geqslant 1 \%$ probability and $L_{\odot}>20$, we are left with five candidates (E1, E2, K1, L1, and N1). These are the most notable stars for further follow up. In addition, a run-away star can be hidden in the general distribution of radial velocities in the direction of SN 1604. This is due to both the very broad distribution of radial velocities (as seen in Figure 4) and the possibility of a high proper motion coupled with a low radial velocity. Obtaining new $H S T$-ACS images would help isolate any associated star by utilizing all three velocity components and enable candidates to be selected to much lower luminosities given the depth of the HST photometry.

Another interpretation is that there are no main-sequence or sub-giant donors in SN 1604. However, there are some caveats to any such statement. In some cases, we extract the spectrum of two or more stars due to their close proximity on the sky the but do not find two radial velocities (except in the extraction aperture Kepler-H), meaning that a star's velocity is missing or that the two radial velocities are indistinguishably close. Possible stars with $L>20 L_{\odot}$ include stars E2 and P2, although with their relative brightnesses compared to the primary star, a large negative radial velocity, consistent with a donor star, should have been visible.

\section{CONCLUSION AND FUTURE WORK}

In this work, we present photometric and spectroscopic observations of candidate stars in the center of the SN 1604 remnant. We can rule out red giant companions due to the lack of bright stars in the field (brightest star Kepler-L with $V=$ 16.5). In addition, we can rule out many candidates in the field that have inconsistent radial velocity signatures (all stars with a $v_{\text {rad }}>0 \mathrm{~km} \mathrm{~s}^{-1}$ have $<1 \%$ probability of being associated with the explosion). Finally, our radial velocity measurements do not identify any peculiar star in this data set beyond what is expected from a Galactic distribution, and are therefore consistent with SN Ia progenitor scenarios that do not leave behind a bright donor star. This finding is similar to work on other historical remnants, such as those which have scrutinized SN 1006 (González Hernández et al. 2012; Kerzendorf et al. 2012a), and the LMC results (Edwards et al. 2012; Schaefer \& Pagnotta 2012).

Both the newly discovered CSM interacting SNe Ia and the lack of donor stars requires an explanation that neither the traditional SD-scenario or the traditional DD-scenario can provide. Justham (2011), Di Stefano et al. (2011), and Hachisu et al. (2012a, 2012b) have suggested a modified SD-scenario in which the companion has time to evolve and become a WD before the explosion of the primary or is intrinsically dim (Wheeler 2012). This can explain the difficulty in finding a stellar companion. If a SN Ia is the result of two WDs merging in a common envelope, this evolution might explain the CSM interaction that is normally not expected for a DD-scenario-it also explains the lack of a companion (Kashi \& Soker 2011; M. H. van Kerkwijk 2013, private communication).

To further test main-sequence and sub-giant progenitor scenarios, additional observations are needed. The current spectroscopic observations only provide an upper limit of $v_{\text {rot }}=$ $200 \mathrm{~km} \mathrm{~s}^{-1}$, which is insufficient to discriminate between unassociated sources with typically not more than a few $10 \mathrm{~km} \mathrm{~s}^{-1}$ and donor stars with $60-140 \mathrm{~km} \mathrm{~s}^{-1}$ (see Figure 4 in Kerzendorf et al. 2012a). We propose high-resolution spectra of the viable stars with $L>10 L_{\odot}(\mathrm{E} 1, \mathrm{E} 2, \mathrm{~K} 1, \mathrm{~L} 1$, and N1) with a multi-object fiber system such as VLT+FLAMES. Such observations would be able to both detect rotation and better detect the blended stars' kinematic signatures. In addition, a single ACS HST follow up observation would allow an accurate measurement of all of the remnant's stars' proper motions, enabling detection of objects with motions consistent with the remnant down to the luminosities expected for even a hot WD.

Currently, only three remnants have been spectroscopically searched for donor stars (SN1572, SN1006, and SN1604), which is a sample plagued by small number statistics. The fourth known Milky Way Type Ia SNR, RCW86, also lends itself to a donor star search. At first glance, this remnant with its lower extinction $\left(A_{V} \approx 1.7\right.$; Leibowitz \& Danziger 1983) and close proximity to Earth $(d=2.5 \mathrm{kpc}$; Williams et al. 2011) seems ideal. However, there are two seemingly separate expansion fronts (one in the southwest and one in the northeast) that would make a center determination very difficult. A safe approach is a search area that encompasses both centers, however, this would involve scrutinizing roughly 2000 stars down to a luminosity of $L=10 L_{\odot}$. This makes RCW86 currently not feasible for a search and thus Kepler is the last remnant easily searched in the Galaxy. In the future, Gaia will measure parallax distances and proper motions, which will enable us to narrow down the possible candidate stars for RCW86.

We thank Ben Shappee for useful discussions on post explosion donor evolution. Furthermore, Steve Reynolds, Kazik Borkowski, Mary Burkey, and Jacco Vink provided crucial points about possible AGB star donors. In addition, we would like to thank Carles Badenes for his description of the literature pertaining to the identity of Kepler's SN. Finally, we thank the anonymous referee for helpful and constructive comments.

J.S. acknowledges the European Research Council for the Advanced Grant Program No. 267399-Momentum.

\section{REFERENCES}

Bandiera, R. 1987, ApJ, 319, 885

Blair, W. P., Long, K. S., \& Vancura, O. 1991, ApJ, 366, 484

Burkey, M. T., Reynolds, S. P., Borkowski, K. J., \& Blondin, J. M. 2013, ApJ, 764, 63

Chiotellis, A., Schure, K. M., \& Vink, J. 2012, A\&A, 537, A139

Di Stefano, R., Voss, R., \& Claeys, J. S. W. 2011, ApJL, 738, L1

Dolphin, A. E. 2000, PASP, 112, 1383

Dopita, M., Hart, J., McGregor, P., et al. 2007, Ap\&SS, 310, 255

Edwards, Z. I., Pagnotta, A., \& Schaefer, B. E. 2012, ApJL, 747, L19

Foley, R. J., Simon, J. D, Burns, C. R., et al. 2012, ApJ, 752, 101

González Hernández, J. I., Ruiz-Lapuente, P., Filippenko, A. V., et al. 2009, ApJ, 691,1

González Hernández, J. I., Ruiz-Lapuente, P., Tabernero, H. M., et al. 2012, Natur, 489, 533

Hachisu, I., Kato, M., \& Nomoto, K. 2012a, ApJL, 756, L4

Hachisu, I., Kato, M., Saio, H., \& Nomoto, K. 2012b, ApJ, 744, 69

Han, Z. 2008, ApJL, 677, L109

Hwang, U., Decourchelle, A., Holt, S. S., \& Petre, R. 2002, ApJ, 581, 1101 Justham, S. 2011, ApJL, 730, L34

Kashi, A., \& Soker, N. 2011, MNRAS, 417, 1466

Katsuda, S., Tsunemi, H., Uchida, H., \& Kimura, M. 2008, ApJ, 689, 225

Kerzendorf, W. E., Schmidt, B. P., Asplund, M., et al. 2009, ApJ, 701, 1665

Kerzendorf, W. E., Schmidt, B. P., Laird, J. B., Podsiadlowski, P., \& Bessell,

M. S. 2012a, ApJ, 759, 7

Kerzendorf, W. E., Yong, D., Schmidt, B. P., et al. 2012b, ApJ, 774, 99

Leibowitz, E. M., \& Danziger, I. J. 1983, MNRAS, 204, 273

Liu, Z.-W., Pakmor, R., Roepke, F. K., et al. 2013, A\&A, 554, 109

Marietta, E., Burrows, A., \& Fryxell, B. 2000, ApJS, 128, 615

Pan, K.-C., Ricker, P. M., \& Taam, R. E. 2012, ApJ, 760, 21

Patat, F., Chandra, P., Chevalier, R., et al. 2007, Sci, 317, 924

Patnaude, D. J., Badenes, C., Park, S., \& Laming, J. M. 2012, ApJ, 756, 6

Podsiadlowski, P. 2003, Astrophysics, arXiv:astro-ph/0303660 
Powell, M. J. 1964, CompJ, 7, 155

Pskovskii, Y. P. 1971, SvA, 14, 798

Reynolds, S. P., Borkowski, K. J., Hwang, U., et al. 2007, ApJL, 668, L135

Reynoso, E. M., \& Goss, W. M. 1999, AJ, 118, 926

Riess, A. G., Macri, L., Casertano, S., et al. 2011, ApJ, 732, 129

Robin, A. C., Reylé, C., Derrière, S., \& Picaud, S. 2003, A\&A, 409, 523

Ruiz-Lapuente, P., Comeron, F., Méndez, J., et al. 2004, Natur, 431, 1069

Schaefer, B. E., \& Pagnotta, A. 2012, Natur, 481, 164

Shappee, B. J., Kochanek, C. S., \& Stanek, K. Z. 2013, ApJ, 765, 150
Simon, J. D., Gal-Yam, A., Gnat, O., et al. 2009, ApJ, 702, 1157

Skrutskie, M. F., Cutri, R. M., Stiening, R., et al. 2006, AJ, 131, 1163

Sollerman, J., Ghavamian, P., Lundqvist, P., \& Smith, R. C. 2003, A\&A, 407, 249

Sternberg, A., Gal-Yam, A., Simon, J. D., et al. 2011, Sci, 333, 856

Wang, B., \& Han, Z. 2012, NewAR, 56, 122

Wheeler, J. C. 2012, ApJ, 758, 123

Williams, B. J., Blair, W. P., Blondin, J. M., et al. 2011, ApJ, 741, 96

Williams, B. J., Borkowski, K. J., Ghavamian, P., et al. 2013, ApJ, 770, 129

Zacharias, N., Monet, D. G., Levine, S. E., et al. 2005, yCat, 1297, 0 